\title{
Stroke diagnosis using HINTS in patients presenting with acute vestibular syndrome: a systematic review

Kailash Krishnan ${ }^{1}$; Kerolos Bassilious ${ }^{1}$; Erik Eriksen²; Philip M Bath ${ }^{1}$; Nikola Spriggi ; Sigrun Kierulf Brækken ${ }^{2}$; Hege Ihle-Hansen ${ }^{3}$; Else Charlotte Sandset ${ }^{2}$

${ }^{1}$ Stroke, Division of Clinical Neuroscience, University of Nottingham and Nottingham University Hospitals NHS Trust, Nottingham, UK; ${ }^{2}$ Department of Neurology, Oslo University Hospital, Oslo, Norway;

${ }^{3}$ Department of Geriatrics, Oslo University Hospital, Oslo, Norway

\section{BACKGROUND}

- Patients with posterior circulation stroke presenting with acute vestibular syndrome (AVSvertigo, nausea/vomiting and gait unsteadiness) represent a clinical dilemma with minimal clinical signs at the time of presentation.

- NIHSS is not reliable and CT scans cannot rule out acute posterior circulation ischaemic stroke.

- The Head Impulse- Nystagmus- Test of Skew (HINTS) test with any of three signs: normal horizontal head impulse, nystagmus that changes direction with gaze, and skew deviation of the eyes is suggestive of a central cause including acute stroke.

- This systematic review examined the effectiveness of abnormal HINTS in posterior circulation stroke in patients presenting with AVS.

\section{METHODS}

- We searched MEDLINE (1966 to 21 December 2017), EMBASE (1980 to December 2017), Web of Science and scanned bibliographies of relevant articles to identify studies on clinical features and diagnostic evaluation.

- Two review authors independently screened and identified articles and resolved discrepancies by discussion to reach consensus.

- We included studies in which HINTS was done primarily to identify posterior circulation stroke with diagnosis (infarct or haemorrhage) confirmed using magnetic resonance imaging (MRI).

\section{RESULTS}

- Six studies (644 patients) with acute vestibular syndrome were identified.

- Two studies had patients with ABCD2 score of 4 and one study examined the relationship between truncal ataxia and posterior circulation stroke.

- The majority of patients in one study had no symptoms at the time of examination.

- Acute stroke was confirmed in 218 patients $(29.8 \%)$.

\section{RESULTS}

- There was a 11-fold increased risk in posterior circulation stroke in patients with a positive HINTS test compared to those with no abnormality (O.R. $11.50,95 \%$ CI 3.50-38.32).

- For any stroke, the pooled sensitivity using HINTS was $93.6 \%$ (95\%CI 90.3-97.0\%) and specificity was $71.6 \%$ (95\%CI $67.4-75.8 \%)$.

- The overall positive predictive value was $60.4 \%$ (95\%CI 55.1-65.8\%) and negative predictive value was $96.0 \%$ (95\%CI $93.9-98.1 \%)$.

- In 2 studies, a positive HINTS identified stroke with greater accuracy than conventional MRI up to 48 hours after symptom onset.

- In one study, HINTS significantly outperformed the ABCD2 score in detecting stroke in AVS.

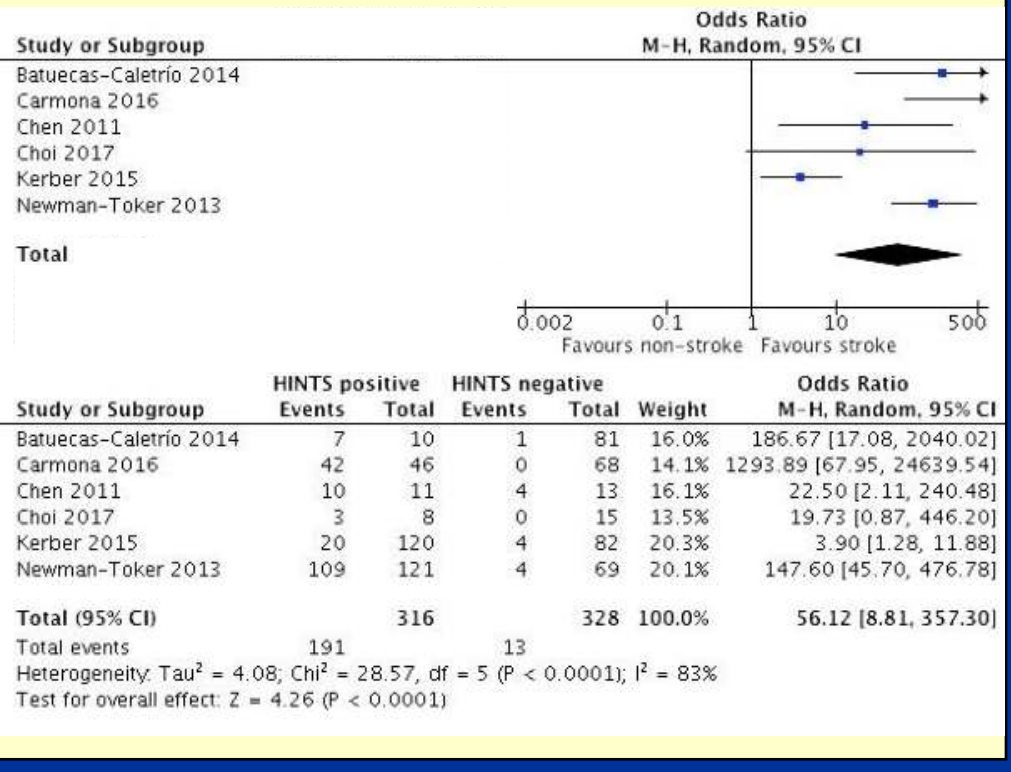

\section{CONCLUSION}

- The data suggest that the HINTS test seems to be able to differentiate posterior circulation stroke from peripheral causes in AVS.

- Since posterior circulation stroke has the same risk of poor outcome as those of anterior circulation, methods to use HINTS in emergency settings need further investigation. 